Fecha de recepción: diciembre 2018 Fecha de aceptación: febrero 2019 Versión final: mayo 2019

\section{Innovación sustentable. Diálogo entre la Ciencia de los Materiales y el Diseño de Industrial}

Javier Alejandro Bazoberri *

Resumen: Mejorar la comunicación del Diseño Industrial con la Ciencia de los Materiales representa una acción necesaria no solo en cuanto a la disposición de información técnica inteligible para el campo del diseño, sino en su intervención directa en la investigación aplicada de nuevos materiales. El perfil socio/económico actual exige a las empresas resolver productos con sensaciones cada vez más específicas y con mayor respeto al medio ambiente. La aplicación regional del modelo de cuatro hélices con la incorporación de la experiencia de usuario presenta ventajas en la interrelación de grupos interdisciplinares y fundamentalmente enfoca la atención hacia problemas socio/ambientales regionales.

Palabras clave: Materiales - Diseño Industrial - Economía Circular - Modelo de Cuádruple Hélice - Experiencia de Usuario - innovación

[Resúmenes en inglés y portugués en la página 42]

${ }^{(*)}$ Diseñador Industrial, graduado en la Universidad Nacional de Mar del Plata. Actualmente auxiliar en la cátedra de Economía y Marketing de la carrera de Diseño Industrial de dicha Universidad. Becario doctoral, bajo el proyecto "Base de datos de perfil ambiental de materiales como aporte a estrategias de diseño sustentable para el sector productivo de Gral. Pueyrredon" y Doctorando por la Universidad de Buenos Aires bajo la Tesis "Metodología interdisciplinar para el desarrollo de nuevos materiales".

\title{
Introducción
}

En los últimos años la legislación argentina ha sancionado una serie de Leyes y Normativas enfocadas hacia mejoras ambientales. Tales obligaciones requieren una respuesta de la sociedad en su conjunto (funcionarios, empresarios, académicos y usuarios). La problemática en el mecanismo de prohibiciones u obligaciones es que pueden generar conflictos quizás aún mayores. Por ejemplo, la emergencia en reducir los residuos plásticos en el ambiente ha impulsado a legisladores a generar mecanismos de prohibición en el uso de este material, provocando la pérdida de puestos laborales en el sector industrial del plástico. Se observa que este tipo de conflictos lleva a diferentes actores a encontrar soluciones integrales, que abarcan desde la concepción de un nuevo material hasta la planificación 
del fin de vida de un producto. El análisis de los plásticos biodegradables permite entender lo que a nivel teórico modelizan el Triángulo de Sábato o de Triple Hélice, donde se postulan relaciones entre Estado, Academia e Industria y lleva a vislumbrar distintas estrategias débiles que se efectúan en la región. Acorde a las limitaciones del modelo de triple hélice y su evolución hacia uno de cuádruple hélice se analiza el rol del diseño y de los ciudadanos como agentes activos, proveedores de información referida a experiencias y necesidades, reafirmados en metodologías experimentales basadas en la Experiencia del Usuario. Se interpreta entonces un escenario donde pueden mejorarse nuevos desarrollos interdisciplinares centrados en el usuario bajo el concepto de economía circular de materiales y productos.

\section{El Estado}

El Derecho Ambiental promueve que la sustentabilidad de los ecosistemas se convierta globalmente en uno de los principios de la ley (Gaines, 2014) y se rige fundamentalmente por la Sustentabilidad Ecológica (Westerlund, 2008). En Argentina se destacan planes estatales que van en cuatro direcciones fundamentales, estrategias para la preservación de bosques y suelos, conservación de biodiversidad, cambio climático y gestión de residuos. Particularmente la gestión de residuos regulada por la Ley de Presupuestos Mínimos 25916 (2004) es obligatoria para provincias y municipios. Está planteada desde el enfoque de la economía circular, cuyo fin es conservar y mejorar el capital natural y optimizar el uso de los recursos, a partir del control de las existencias finitas (recirculación de productos, componentes y materiales) y el equilibrio de flujos renovables (Ellen McArthur Foundation, 2013). El Plan Estratégico Provincial (en adelante PEP) a cargo de las provincias, es un instrumento que al ser aplicado en los distintos municipios pretende efectivizar esta Ley en cualquier punto del país. El concepto de economía circular o de "residuo como insumo" plantea además en el PEP, temáticas transversales como producción más limpia y consumo responsable, por lo que involucra tanto al sector privado (insumos, fabricación y logística) y a los consumidores o ciudadanos. Dentro de las obligaciones que se encuentran delimitadas en el PEP se encuentran objetivos de interés como la disminución de la cantidad de residuos a disposición final y promover políticas de prevención, reutilización, minimización y reciclaje a largo plazo. Estos primeros objetivos impulsan por ejemplo en la Provincia de Buenos Aires, la sanción de normativas obligatorias a partir de la Ley provincial de Gestión Integral de Residuos Sólidos Urbanos N. 13657 (2006), otorgando fondos, programas de reducción, reutilización y reciclaje, concursos, y generando prohibiciones. Es en estas normas complementarias donde se encuentra una articulación problemática. La única Ley complementaria prohibitiva a la de gestión de residuos es la Ley N. ${ }^{\circ} 13868$. Establece prohibir en toda la provincia, el uso de bolsas de polietileno y todo material plástico convencional para transporte de productos o mercaderías, afectando no solo a los productores sino también a los proveedores y distribuidores. El concepto que modeliza claramente esta situación es el de las articulaciones del desarrollo sustentable (Mulder et al, 2011). Una articulación es la materialización de una motivación, que puede desencadenar sucesos positivos o negativos en el futuro. Es decir, una articulación legal 
generada a partir de una motivación por el cuidado ambiental provoca un perjuicio en el ámbito productivo. Este escenario puede impactar positivamente, como en la valorización de la investigación y desarrollo de nuevos materiales e insumos sustitutivos, como también de forma negativa y abrupta en las distintas economías del sector del plástico y en consecuencia en los hábitos de consumidor.

\section{La Investigación y el Desarrollo de nuevas soluciones.}

Como caso de estudio, los materiales sintéticos pueden explicar la relevancia de la ciencia de los materiales sobre el desarrollo sustentable y el diseño de productos. La paradoja en la invención de los polímeros sintéticos es que parten de una necesidad relacionada con la sustentabilidad. En 1863 la empresa estadounidense Phellan \& Collander ofrecía diez mil dólares a quien pudiera sustituir el marfil en las bolas de billar, con la urgencia de conseguir un material más barato y de fácil obtención, que en perspectiva determinara una reducción considerable en la caza furtiva de elefantes (Sosa, 2003). Es así como surge el celuloide que, si bien no ganó el premio ya que las bolas al chocarse explotaban, establecieron antecedente en una investigación posterior, el primer plástico sintético termoestable inventado en 1909 por el químico Leo Baekeland, llamado "bakelita", y utilizado ampliamente en carcasas de teléfonos, radios, artículos de oficina y de hogar (García, 2009). Tanto este primer invento como los que surgieron posteriormente (Nylon, PVC, Policarbonato, Poliestireno, entre otros) determinaron el auge de materiales artificiales adoptados por bienes de amplia aceptación social (Ashby, 2009; Karana et al., 2014). Sin embargo, el uso masivo de los plásticos desató múltiples problemáticas ambientales debido a la necesidad de explotación de restos fósiles y su difícil degradación, afectando principalmente a la biodiversidad terrestre y marina. La realidad ambiental global y nacional demandan entonces una nueva revisión sobre el desarrollo de los materiales sintéticos dentro de la complejidad actual en esta industria.

A partir de la experiencia del plástico como insumo gestado en un laboratorio, la cadena global de valor en de esta industria comienza a dividirse según las fases de investigación y desarrollo (academia), introducción al mercado (industria) y aceptación social (ciudadanía) (Ashby \& Johnson, 2002; Manzini, 1986; van Keresten \& Kandachar, 2004). Tres eslabones que dependiendo de la región requieren de un cuarto agente, el Estatal. A diferencia de los países centrales, la inversión en Ciencia y Tecnología en Latinoamérica se sustenta por parte del Estado. En Argentina aproximadamente el 78 \% de la inversión en Investigación, desarrollo e innovación (en adelante $\mathrm{I}+\mathrm{D}+\mathrm{i}$ ) es pública, mientras que en Estados Unidos el $71 \%$ de la inversión en I+D+i es privada (UNESCO, 2015). De todas formas, el patentamiento no sólo depende de la necesidad de captar determinados mercados, sino que forma parte de una idiosincrasia propia de cada nación para proteger sus invenciones. Es de destacar para este caso, que en Argentina mediante una búsqueda de las principales patentes solicitadas (Patentscope - WIPO 2008-2017), se encuentran en primer lugar el tipo C12N (Química, Bioquímica, microorganismos o enzimas; propagación, cultivo o conservación de microorganismos; técnicas de mutación o de ingeniería genética y medios de cultivo) y en tercer lugar el campo B65D (transporte; embalaje; al- 
macenado; manipulación de materiales delgados o filiforme, recipientes para el almacenamiento o el transporte de objetos o materiales). Los indicadores anteriores representan una fortaleza del sector químico y bajo este esquema una oportunidad para el desarrollo de envases y recipientes a base de materiales inteligentes (biotecnología-nanotecnología). Sin embargo, que existan desarrollos en el área, no significa que respondan a la Ley de prohibición N. ${ }^{\circ} 13868$. ¿Qué se encuentra entonces sobre el desarrollo de nuevos materiales o envases, para el transporte de productos o mercaderías bajo el enfoque de economía circular? Según el Portal de Información de Ciencia y Tecnología Argentino dependiente de la Secretaría de Ciencia y Tecnología de la Nación, los proyectos encontrados bajo las etiquetas "materiales" y "envases" en el área de "ciencia", se radican en Buenos Aires y C.A.B.A en un $71 \%$, y el resto se reparte entre las provincias de Córdoba, Santa Fe y Entre Ríos de forma equitativa, casi el $90 \%$ corresponde a proyectos de economía circular. Con una inversión pública (FONCYT - CONICET) de U\$D 770677 (2010-2015) se destacan en la región el Centro de Investigaciones en Criotecnología de Alimentos (CIDCA) (U\$D 319048) ubicado en La Plata y la División de Ecomateriales del Instituto de Investigaciones en Ciencia y Tecnología de Materiales (INTEMA) (U\$D 284137) ubicado en Mar del Plata. Tal financiamiento en ambas instituciones corresponde a proyectos relacionados con la economía circular. La primera con una mirada en la aplicación de materiales biodegradables en el sector agroindustrial, envases alimenticios y encapsulados, donde participan profesionales del área de Química, Agronomía, Alimentos y Cs. Biológicas. Y la segunda que además tiene líneas en hidrogeles, nanocompuestos, bioespumas, bioadhesivos, construcción y bioenergía, donde participan principalmente Ingenieros Químicos, en Materiales, en Alimentos y Mecánicos. Los principales resultados se verifican en la instancia de patentamiento internacional (Patentscope - WIPO, 2018) con las denominaciones AR080876 (CIDCA - UNLPA - CONICET) Película biodegradable, procedimiento para su preparación y usos. Y la AR075002 (INTEMA - UNMDP/UBA - CONICET) Material biodegradable biocompatible y no tóxico, para aplicación en productos alimenticios, farmacéuticos, cosméticos y de limpieza. Se detecta además un proyecto colaborativo entre Academia e Industria (AR107134) con participación de CONICET - UBA (2016) para fabricar envases o bolsas biodegradables que puedan contener, y transportar productos de diversos tipos.

\section{El modelo de Triple Hélice y sus limitaciones.}

El modelo de Triple Hélice (Etzkowitz y Leydesdorff, 2000) plantea múltiples esquemas de interacción para sus integrantes, conocidos como actores o esferas institucionales similar al Triángulo de Sábato (Sábato y Botana, 1968), aunque con distintas etapas evolutivas. En la primera etapa el Estado abarca la Industria y la Academia, regula las relaciones entre las esferas institucionales, por lo que puede verse muy similar al triángulo de Sábato. Suele convivir en países con esquemas políticos socialistas donde el Estado ejerce un importante papel en el sector industrial. En la segunda etapa se separan las esferas institucionales y se circunscriben las relaciones entre ellas, en este caso existen fuertes barreras entre actores. En la tercera etapa la infraestructura de conocimiento queda en función de la interrela- 
ción de las esferas institucionales donde entran en juego los entes híbridos. Se identifican tres tipos de entes híbridos: Start Up, Spin Out, Spin off, en general son agencias pequeñas e intermedias que no entran en la clasificación de Gobierno, Empresas, Universidades. A primera vista pareciera que el modelo de interacción entre Estado a partir de financiamiento, la producción académica concretada en patentamientos y la garantía de las empresas a generar desarrollos vinculantes, funciona de forma coherente en el caso de los plásticos biodegradables. Sin embargo, debe observarse detenidamente que este caso puede enmarcarse en la segunda etapa de este modelo. Los convenios firmados entre empresas privadas y laboratorios públicos, y colaboraciones entre ambas partes, tienden a dilatarse en el tiempo principalmente por la necesidad de presupuesto en el desarrollo de plantas piloto y adaptación de las patentes a un producto particular, lo que lleva a los laboratorios públicos argentinos no solo a investigar los insumos sino también a promocionar las patentes a partir de potenciales aplicaciones o productos. En la actualidad argentina, todavía no existe un envase biodegradable fabricado 100\% en la región, desde su insumo hasta el producto final. Los laboratorios deben convivir con un escenario donde existe una alta competitividad de cuatro grandes multinacionales proveedoras de insumos biodegradables, principalmente europeas, y una negativa de empresas extranjeras para desarrollar trabajos colaborativos con el sector público debido a una baja rentabilidad en comparación a otros países con mayor consumo de este material.

El único modelo principal de interacción que funcionó a partir de la Ley de prohibición de bolsas tradicionales es mediante la colaboración entre agentes privados, locales y extranjeros que se puede entender como modelo tradicional de innovación sumado al apoyo Estatal en cuanto a la posibilidad de importar tales insumos. Empresas de I+D+i extranjeras y fabricantes de bolsas locales trabajan con el fin de acelerar el desarrollo productivo local a fin de acatar esta ley. Actualmente 19 empresas se encuentran habilitadas bajo el Registro de Fabricantes, Distribuidores, e Importadores de Bolsas Para el Transporte de Mercaderías, dependiente del Organismo Provincial Para el Desarrollo Sostenible (2018). Dicha habilitación se obtiene a partir de un estudio realizado en el Centro de Biodegradabilidad y Compostabilidad de Plásticos impulsado por UBATEC S.A (2018). Sin embargo, el resultado de certificaciones refleja un nivel muy bajo de readaptación de la industria local siendo que la ley ya lleva 10 años sancionada. Durante el período 2017 - 2018 los controles en la aplicación de la Ley se han intensificado y con ello se evidencian turbulencias en el sector. Si bien la readaptación se genera a partir de un cambio en el insumo y una nueva calibración en las máquinas sopladoras, los insumos biodegradables son en la actualidad tres veces más caros que los no degradables. Este impacto puede ser absorbido por grandes empresas que demuestran ser en un 70\% la mayoría de las empresas habilitadas. Sólo un $30 \%$ empresas de menos de 25 empleados han podido acatar esta normativa y evidencia una grave problemática. Un análisis realizado por la Cámara Argentina de la Industria Plástica (CAIP), la Asociación Civil Ecoplas y el Sindicato del Plástico en la Ciudad Autónoma de Buenos Aires (C.A.B.A) muestra que la prohibición parcial que se da en el año 2017, y la total en el 2018 con la Resolución 341/16/APRA en C.A.B.A presenta una caída del 75\% de producción, pérdida de 700 puestos laborales directos, 2400 indirectos y caída del empleo del $24 \%$. Las empresas perjudicadas son las Pymes, empresas de 1 a 25 empleados que no cuentan con la posibilidad de reconversión y que deriva en el cierre de 50 empresas en 2017 (Pilar Productivo, 2018). 
En este escenario cabe hacer un paréntesis ya que desde el año 2013 al 2016, ante la imposibilidad de satisfacer la demanda por la sanción de esta ley, se aprobó la creación de la norma IRAM N. 13610 en conjunto con la Cámara Argentina de la Industria Plástica (CAIP), la Asociación Civil Ecoplas y el Sindicato del Plástico en la Ciudad Autónoma de Buenos Aires (C.A.B.A). Establecieron junto con el Ministerio de Ambiente de la C.A.B.A un cambio de estrategia para la reducción de residuos plásticos cambiando el sistema gratuito de bolsas por la venta de un tipo de bolsa plástica más grande, más resistente, con los colores negro y verde para fomentar su reutilización como bolsa de residuo y el reciclaje a partir de sus códigos de color. Otras estrategias conjuntas como campañas verdes cercanas a los domicilios, puntos verdes en las plazas y políticas públicas de difusión orientada a los ciudadanos lograron reducir un $70 \%$ el consumo de bolsas plásticas. Esta estrategia si bien no significó el acatamiento de la Ley impulsada por Nación, obtuvo una reducción del $70 \%$ en el consumo de bolsas, y favoreció la clasificación de residuos en origen. Contrariamente a partir del control de la Ley en 2016, por la variación del suministro de bolsas negras y verdes de forma habitual se provocó una distorsión en la separación de residuos en origen, por lo que por ejemplo en el ámbito porteño por ejemplo "40.000 toneladas de polietileno no lleguen a los recicladores" (CAIP, 2016), y en este sentido las plantas recicladoras cuenten con un 50\% de capacidad ociosa Con este apartado cabe reflexionar que la problemática en la prohibición no sólo llevaría efectos negativos al sector empresarial, sino también al hábito de los ciudadanos, ya que al no prever que las empresas se verían dificultadas de abastecer rápidamente con bolsas biodegradables, los ciudadanos se encontrarían con menores facilidades para la separación de residuos.

El Gráfico 1 permite visualizar los tres modelos desarrollados, donde el primero no fomenta el desarrollo científico local y perjudica la sustentabilidad de las empresas más pequeñas. En el segundo modelo se presenta dilatación temporal y las empresas privadas no invierten en el desarrollo piloto de patentes nacionales. El tercer modelo alternativo que no acata la normativa presenta una posible interacción entre agentes con ventajas orientadas hacia el hábito de los ciudadanos, y parece funcionar de forma parcial. En este sentido se debe posibilitar el pasaje gradual de grandes y pequeñas empresas hacia una producción sustentable, mediante estrategias impulsadas desde el Estado y la Academia para seducir a la Industria y fundamentalmente darle respuestas al ciudadano común. Se apoya la teoría donde la sociedad moderna está enfrentada a problemas con una complejidad caracterizada por la imposibilidad de ser resueltos a partir del conocimiento existente (Kuhn, 1971) y donde sistemas complejos como los que aborda la ciencia post-normal pueden adecuarse al desarrollo sustentable de materiales y productos (Ashby, 2015). La ciencia post-normal comprende problemas donde los hechos son inciertos y las decisiones urgentes. La incertidumbre ronda la ignorancia, por lo tanto, adquiere peso el consenso público y la participación de todos los que están asumiendo el riesgo (Natenzon, C. 2003). Análogamente los últimos sistemas de innovación como el de cuádruple hélice demarcan la necesidad de incorporar a los propios "usuarios" o "ciudadanos" en los laboratorios y en proyectos de $\mathrm{I}+\mathrm{D}+\mathrm{i}$. 


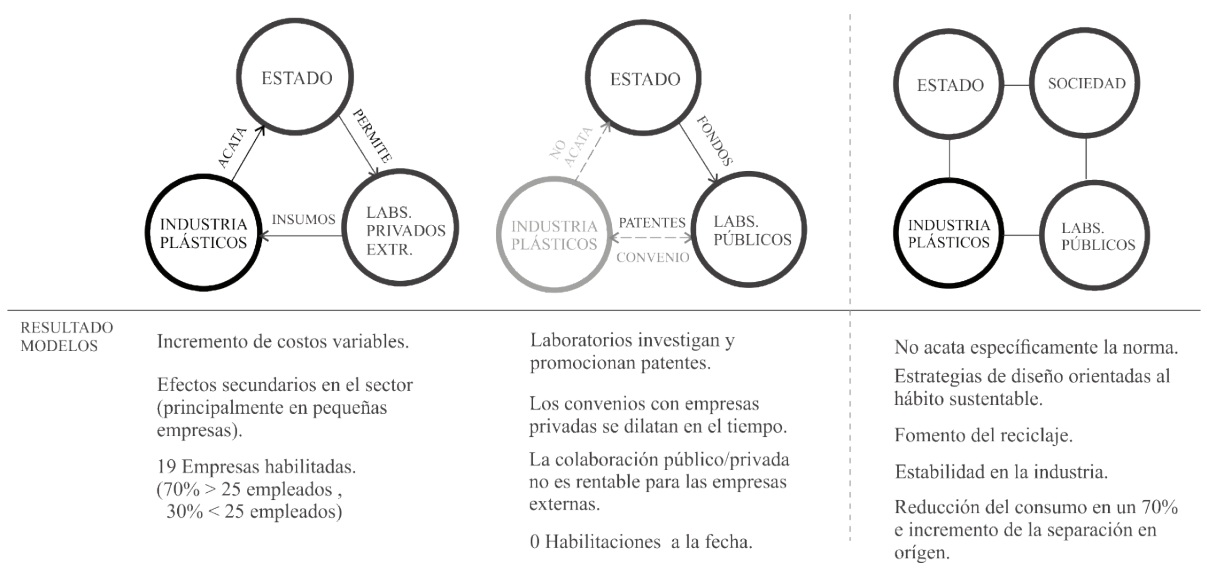

Gráfico 1. Relaciones generadas a partir de la Ley 13.868. Elaboración propia.

\section{Del modelo de Triple Hélice al de Cuádruple Hélice Los usuarios y el diseño.}

El modelo de Cuádruple hélice (Arnkil et al, 2010), incorpora además de los actores de la Triple Hélice a la Sociedad como agente para la innovación. Existe una discusión en esta evolución ya que se interpreta que la sociedad ya se encuentra involucrada dentro de los tres actores iniciales y que estas interrelaciones beneficiarían a la sociedad dentro de la que se desenvuelven. Sin embargo, se identifica en el modelo de la Cuarta Hélice que la Sociedad tracciona desde el rol de Usuarios. Y en este sentido se entiende que para un proyecto particular no cualquier ciudadano es Usuario de un diseño determinado. Por lo que el conjunto de Usuarios con características similares tendientes hacia la adquisición de un producto resulta de gran interés para cualquier proyecto de innovación. El modelo tradicional de cuádruple hélice es similar al modelo de triple hélice excepto por la recopilación sistemática y la utilización de la información del usuario. El foco está puesto en el desarrollo de innovaciones comerciales de alta tecnología basadas en los últimos conocimientos de investigación científica. El propietario del proceso de innovación es una empresa, un grupo de empresas, una universidad o un grupo de universidades. En este caso los usuarios son tratados como informantes, no como desarrolladores. 


\section{La ciencia de los materiales, diseñadores y usuarios.}

La elección del usuario como cuarto candidato se ve respaldada por las opiniones presentadas en recientes investigaciones y políticas de innovación donde ya no se pueden confiar en mantener una ventaja competitiva basada en factores "tradicionales" de precio y calidad. Las empresas se ven forzadas a buscar fuentes alternativas de ventaja competitiva y, por lo tanto, están llevando a cabo transformaciones importantes en sus procesos de innovación y modelos comerciales para entregar productos y servicios más valiosos al mercado. Esto implica modelos comerciales cada vez más abiertos, un mayor enfoque en la comprensión de las necesidades latentes de los consumidores y una participación más directa de los usuarios en las diversas etapas del proceso de innovación. No quita claramente que los modelos puedan convivir. A diferencia del modelo de Triple Hélice que parece enfocarse en producir innovación de alta tecnología a partir del peso depositado en los agentes académicos, el modelo de Cuádruple Hélice puede verse como una forma sistemática de buscar innovación orientada al usuario. Particularmente en este problema de investigación es conveniente reforzar la fuerte correspondencia en términos proyectuales que tienen los materiales diseñados por empresas químicas privadas en comparación con el diseño de productos industriales ligados a satisfacer un segmento de consumo. Comenzando por la especificación de distintas necesidades, se piensan en ideas y alternativas, se selecciona la más adecuada y finalmente se decide qué forma debe tomar el producto y cómo se puede fabricar. Es la incorporación del proceso habitual de diseño en productos donde la concepción parte desde la generación de un material o sustancia específicos, presentes en protocolos utilizados en el "Chemical Product Design" o "Diseño de Productos Químicos" en empresas como Motorola, W. L. Gore y DuPont (Moggridge y Cussler, 2000, p.7). El planteo introducido por Hassenzhal (2010) define además que el desarrollo de materiales abordado por satisfacciones funcionales y necesidades hedónicas debe ser estudiado de manera interdisciplinar, como el caso Ducht Design Meets Bamboo (van der Lugt, 2008), que presenta la importancia en la transformación de materiales renovables como estrategia fundamental para el diseño sustentable. Particularmente valoriza la alta tasa de crecimiento del bambú, sus cualidades estructurales y fácil procesamiento. El estudio de van der Lugt expone la dificultad de adaptar dicho material al diseño de productos en Europa Occidental, se centra en el análisis de la cadena de valor de los materiales e identifica principalmente la dilatación temporaria que se produce en las fases de introducción al mercado y aceptación por la baja capacidad productiva y preconceptos estéticos del bambú. Afirma que es necesario convencer a todos los miembros relevantes de la cadena de valor antes de que un material pueda llegar finalmente al mercado de consumo masivo. Afirma bajo circunstancias particulares que los diseñadores pueden funcionar como líderes de opinión debido a su posición clave en la cadena de valor de la mayoría de los bienes duraderos de consumo. Estos vinculan a los productores de materiales y fabricantes, con los potenciales consumidores finales al traducir las oportunidades que plantea un material, en un producto comercial concreto. Concluye que para los productores de material de mediana / baja escala activos en el sector de los bienes de consumo duraderos de mediano a alto nivel, las intervenciones de diseño pueden actuar como un instrumento 
importante para desarrollar el conocimiento, aumentar la conciencia, generar exposición y como resultado estimular su tasa de comercialización.

Particularmente el concurso de innovaciones de mayor relevancia en la República Argentina llamado INNOVAR convoca anualmente a científicos, diseñadores y emprendedores de todo el país conglomerando no solo instituciones públicas, sino también al sector privado y a jóvenes emprendedores. Dentro de las distintas convocatorias, en el campo de los nuevos materiales se encuentran diseñadores que mediante la búsqueda de innovación desarrollan nuevos materiales, y científicos que mediante la necesidad de promocionar sus desarrollos diseñan nuevos productos. En la Gráfica 2. se puede observar un muestreo que analiza desarrollos pertinentes en el área de materiales basados en la economía circular contemplando nuevos materiales y compuestos (INNOVAR, 2014-2018). Puede observarse una tendencia creciente, donde las disciplinas relacionadas al diseño concretan la mayoría de los proyectos principalmente con ideas basadas en el reciclaje y reutilización de desperdicios de distintas industrias. En cuanto a los laboratorios se encuentran presentaciones en un numero más reducido pero que tienen que ver principalmente con nuevos desarrollos desde la caracterización química.

\section{MATERIALES - INNOVAR (2014-2018)}

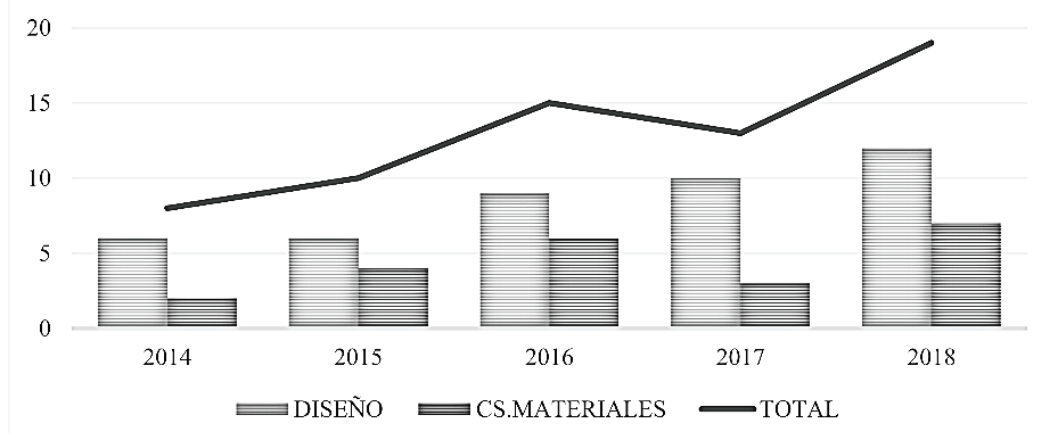

Gráfico 2. Muestreo de proyectos en relación con materiales sustentables. Elaboración propia.

Ambos campos disciplinares generan materiales y productos para nichos particulares. Cabe entonces realizar una comparación que alude y refuerza el concepto de la necesidad de trabajo interdisciplinar bajo el modelo de Cuádruple Hélice. Según este muestreo se ha realizado una evaluación a partir del concepto de Experiencia del Usuario de Jordan (2000), donde se analizan productos bajo aspectos funcionales, de usabilidad y de satisfacción (estética, asociaciones y percepciones). Con una ponderación de 1 a 5 se han eva- 
luado los desarrollos en laboratorios (Gráfico 3) y aquellos proyectados por diseñadores (Gráfico 4). Se encuentran proyectos análogos principalmente en productos del sector de la construcción (ladrillos, tejas, aislantes) y descartables (macetas y utensilios). Se observa que el concepto de satisfacción en los productos ingenieriles tiende a descender con respecto a los generados por diseñadores, principalmente las pautas de transformación formal, uso de colores y texturas, que derivan en asociaciones sobre un público particular. Sin embargo, ambos campos trabajan considerablemente estos conceptos. Luego se destaca que la usabilidad de los productos en el caso de productos de ingeniería tiende a valorizarse más que al de satisfacción. En los productos generados por diseñadores existe una clara correlación entre la usabilidad del producto y la relación entre el trabajo estético/sensorial.

\section{INGENIERÍA de MATERIALES Y PRODUCTOS SUSTENTABLES}

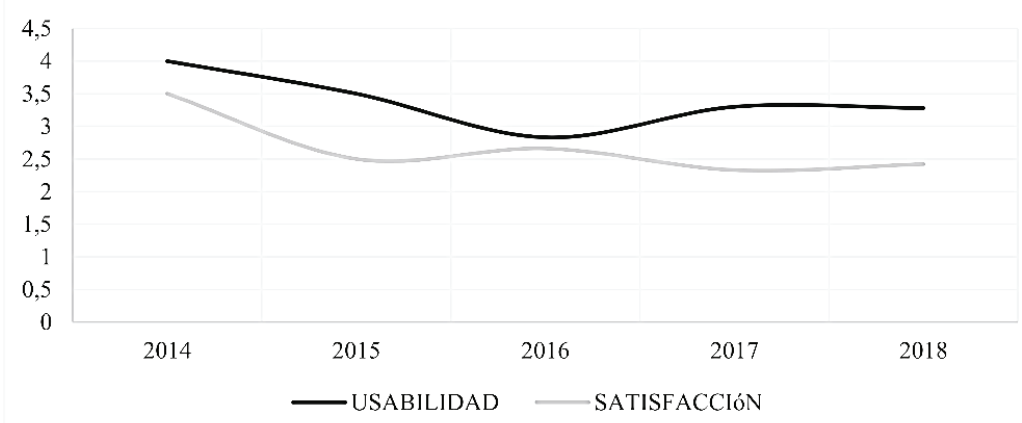

Gráfico 3. Ponderación materiales y productos de laboratorios. Elaboración propia.

\section{DISEÑO de MATERIALES Y PRODUCTOS SUSTENTABLES}

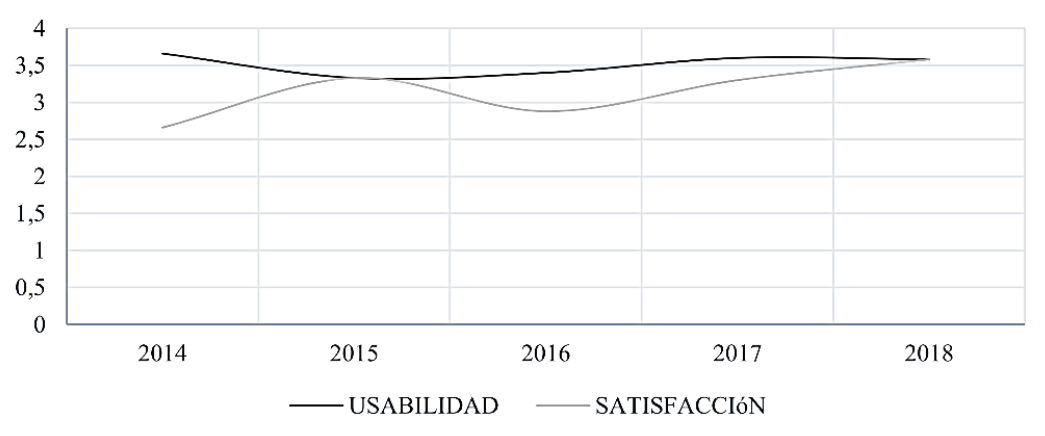

Gráfico 4. Ponderación materiales y productos de Diseño Industrial. Elaboración propia. 
Por lo observado, el perfil socio/económico actual lleva a diseñadores y a científicos a generar propuestas con mayor respeto al medio ambiente y con sensaciones cada vez más específicas. En este sentido se potencian las herramientas prototípicas de desarrollo a partir de la Experiencia del Usuario, que se enfocan en una combinación entre las ciencias físicas (material como materia), la psicología y la fisiología (material como experiencias personales) y las ciencias sociales (materiales como experiencias colectivas y fenómenos culturales). Un método que desprende información sustancial para potenciar las habilidades creativas del diseñador, actuar con mayor sensibilidad ante las necesidades de este entorno y fluidez para el uso de conocimientos propios del campo del diseño (verbales, ideativas, figurativas, semánticas, simbólicas, asociativas y expresivas). Se pretende enriquecer lo que en métodos tradicionales de diseño se identifica como pensamiento divergente o lateral, bajo herramientas como brainstorming, mapas mentales, método de los sombreros, entre otros.

En el Centro de Investigaciones Proyectuales y Acciones de Diseño Industrial (CIPADI), que funciona en la Facultad de Arquitectura, Urbanismo y Diseño en la Universidad Nacional de Mar del Plata, se han llevado a cabo experimentaciones tanto en el ámbito de la educación como en el desarrollo concreto de materiales bajo la metodología de Experiencia del usuario. Desde la enseñanza del marketing en Diseño Industrial esta metodología alienta el análisis estético sensorial del segmento de consumo, en pós de vincular saberes de la ciencia de materiales y el diseño, ya que en diversas ocasiones la generación de valor se establece a partir de la aplicación de nuevos materiales y el valor de la innovación del producto resulta mayormente propio de la novedad del material (Bazoberri y Zimmerman 2018). A su vez en la Investigación se aborda la aplicación de metodologías experimentales para el análisis de la Experiencia del Usuario en el desarrollo de materiales sustentables, con tal de evaluar su pertinencia en el proceso de desarrollo conceptual del producto. Apuestan a la incorporación de la experiencia del usuario para analizar su satisfacción. Tal intervención repercute directamente en la fase de extensión de vida útil del D4S (relación usuario-producto y confiabilidad) y paralelamente permite identificar conductas humanas que tiendan al Desarrollo Sustentable (Canetti y Bazoberri 2018). En el 2015 a mano del departamento de Ingeniería del Diseño en la Universidad Tecnológica de Delft y del Departamento de Diseño del Politécnico de Milano, teóricos como Karana, Barati, Rognoli y van der Laan han desarrollado un método basado en la incorporación de requisitos/necesidades a partir de la experiencia del usuario, llamado Material Driven Design (MDD), esta metodología se basa en el análisis de desempeño sensorial, interpretativo y afectivo acorde al posible consumidor final y cómo éstos se relacionan con las propiedades físicas (técnicas) de un material. Obligan dentro del proceso de desarrollo del material a incorporar requisitos del usuario en base a experiencias detectadas en el transcurso del proceso. Luego de analizar e interpretar los resultados, que revelen las experiencias positivas y negativas del material, el diseñador prevé las intenciones de diseño para la mejora y desarrollo de nuevos materiales. Manifestando los patrones para evocar la experiencia de materiales imaginados, el diseñador crea y materializa conceptos que hacen la transición de la intención de diseño al diseño de material / producto. Este método parte de tres posibles escenarios, diseñar con un material relativamente conocido, encontrando nuevas experiencias en otras áreas; diseñar con un material relativamente 
desconocido, brindándole al diseñador espacio para definir posibles áreas de aplicación y nuevos significados y experiencias; y por último diseñar con una propuesta material, con semi-desarrollos o muestras de testeo (compuestos de comida desechada, materiales vivientes hechos de bacterias, textiles $3 \mathrm{D}$, etc.) definidas a través del proceso de diseño en relación al área seleccionada, generando además retroalimentación para otros materiales en desarrollo. En la actualidad comunidades globales de diseño se encuentran haciendo foco en entender, interpretar e imaginar experiencias planteando nuevas estrategias de innovación a partir de la experiencia del usuario (Verganti, 2009) y particularmente en colaboración con expertos de otras áreas científicas (Miodwnik, 2007).

\section{Factibilidad del diálogo}

Una metodología para la generación de nuevos materiales y productos con perfil interdisciplinar que incluya además la visión del Diseñador en los laboratorios podría resultar una oportunidad para Científicos y Diseñadores argentinos. Un sondeo realizado en el Instituto de Investigaciones en Ciencia y Tecnología de Materiales (INTEMA) mediante una encuesta representativa del 10\% de los profesionales, indica varios puntos de acción en este sentido. Sólo un 30\% llega a generar patentamientos y la mayoría de los desarrollos permanecen en investigaciones aplicadas. Declaran que las posibles demoras entre el desarrollo de un material y la comercialización de un nuevo insumo puede tardar entre cinco a diez años, encontrando la principal demora entre la fase de investigación aplicada, prototipo/patentamiento y planta piloto. Si bien cuentan con trabajo interdisciplinar, un $60 \%$ trabaja entre ingenieros y un $40 \%$ incorporan otras profesiones como licenciados en química, economía y otros. Las principales dificultades que encuentran en este sentido tienen que ver con respetar los plazos de entrega en tareas asignadas, uso de comunicación y terminología adecuada, y la definición de requisitos en común que deriven en una

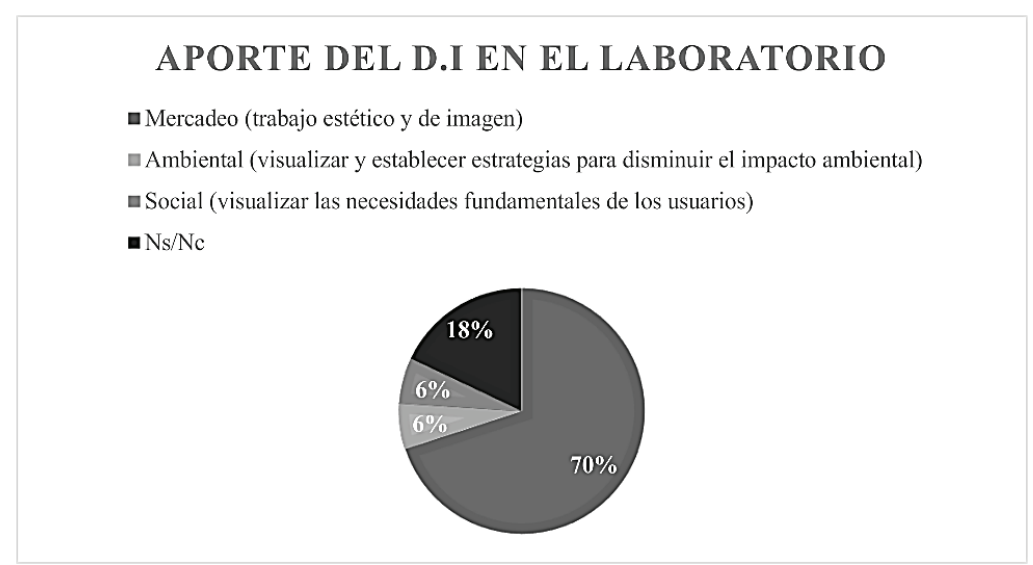

Gráfico 5. Potencial interacción con el DI en laboratorios. Elaboración propia. 
característica del material concreta. No se encuentran trabajos colaborativos con el diseño industrial, aunque se analiza la factibilidad del trabajo en conjunto. Se destaca que la mayoría aceptaría trabajar con diseñadores encontrando mayores beneficios en aspectos comerciales (Gráfico 5) fuertemente relacionados con la Experiencia del Usuario (Gráfico 6), donde entienden que la relación puede darse en cuestiones de seguridad del usuario y aplicación de variables estético/sensoriales.

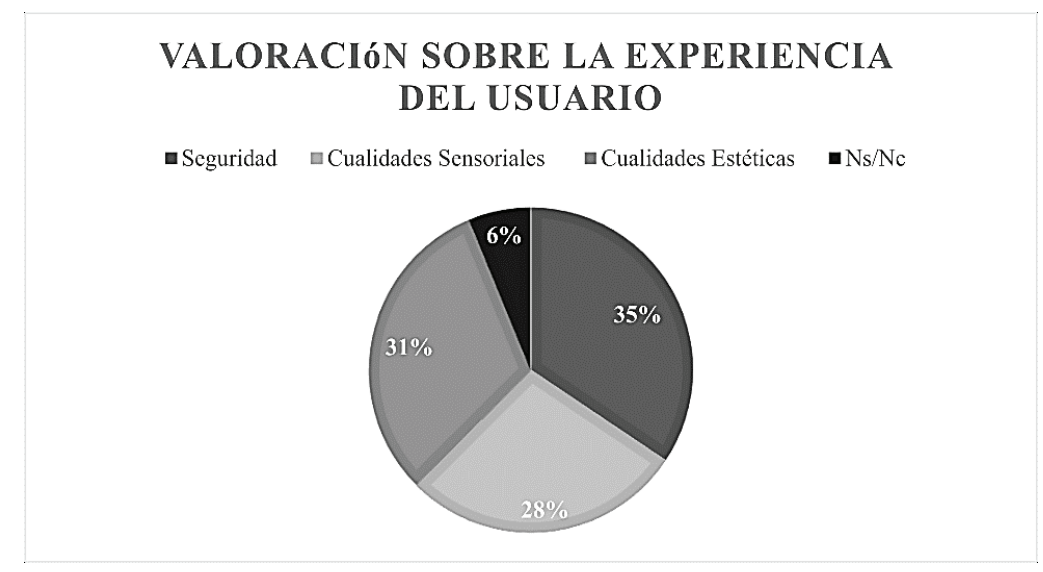

Gráfico 6. Valoración de la experiencia del usuario en laboratorios. Elaboración propia.

\section{Conclusión}

Para interpretar de manera más eficiente las problemáticas y necesidades del entorno sin perjudicar el ambiente y a las sociedades futuras se deben tomar decisiones estratégicas desde el inicio del proyecto interpretando qué articulaciones se producen y cuáles son sus impactos. Una decisión fundamental es la elección del material constitutivo, entenderlo, comprender sus limitaciones y sus oportunidades donde ha quedado en evidencia que aún se debe mejorar la comunicación entre los Científicos y los Diseñadores. El modelo de triple hélice para la innovación científica que involucra tanto al Estado, como a la Industria y a la Academia debe mudar a uno de cuatro hélices que incluya además la opinión del consumidor o usuario. El llamado "Análisis de Experiencia de Usuario" también está involucrado en la caracterización o desarrollo de nuevos materiales y se puede encontrar en modelos de desarrollo de productos donde se permiten generar propuestas de nicho con sensaciones cada vez más específicas y personalizables, que es principalmente donde hoy la Argentina tiene campo de acción. El nivel de desarrollo científico difiere entre países desarrollados y países en vías de desarrollo, principalmente por el tipo y volumen de inversión. Mientras que en los desarrollados existe una mayor inversión del sector 
privado, en Latinoamérica predomina una menor inversión que es inyectada por parte del Estado. En Argentina puntualmente los sectores que permiten estudiar el marco de la investigación, desarrollo, innovación y diseño de nuevos materiales son principalmente instituciones públicas en representación de la Academia, las decisiones del gobierno vigente en representación del Estado, y las distintas industrias relacionadas a los materiales sintéticos. Se debe observar cómo mejorar la interrelación entre el sector privado y académico y la comunicación de grupos interdisciplinarios. Perfilar las investigaciones hacia beneficios socioambientales y estabilizar el apoyo al I+D+i junto con la actuación del Diseño Industrial. Indagar particularmente en la Experiencia de Usuario rompe con la linealidad "Científico - Productor - Diseñador - Usuario/Consumidor" y en este sentido fomentar estrategias integrales para la relación entre sectores público/privados, mejorar la competitividad y generar pautas para un desarrollo socioambiental responsable.

\section{Agradecimientos}

Al Dr. Lorenzo R. Basso, presidente de UBATEC. A las Dras. Silvia Goyanes y Lucía Famá, investigadoras del Laboratorio de Polímeros y Materiales compuestos de la Universidad de Buenos Aires. Al Dr. Facundo Altuna, investigador del INTEMA y a integrantes del Centro de Investigaciones Proyectuales y Acciones de Diseño Industrial (CIPADI), por su colaboración para el desarrollo de la problemática abordada.

A la Dra. Beatriz Galán, quien fuera directora del Centro de Proyecto, Diseño y Desarrollo de la FADU-UBA por su convicción para la generación de atmósferas de pensamiento estratégico y creación, en el desarrollo de proyectos regionales.

\section{Lista de Referencias Bibliográficas}

Arnkil, R., Jarvensivu, A., Koski, P., Piirainen, T. (2010). Exploring Quadruple Helix. Outlining user-oriented innovation models. Tyoraportteja, volumen (85), 1-113.

Ashby, M., Balas, D. F., y Coral, J.S. (2015). Materials and Sustainable Development.3ra edición. Oxford, Reino Unido: Butterworth-Heinemann Elsevier.

Ashby, M., y Johnson, K. (2002). Materials and design. The art and science of material selection in product design 1ra edición. Oxford, Reino Unido: Butterworth-Heinemann Elsevier.

Ashby, M., y Johnson, K. (2009). Materials and design. The art and science of material selection in product design 2da edición. Oxford, Reino Unido: Butterworth-Heinemann Elsevier.

Bazoberri, J., Zimmermann, M. (agosto de 2018). El diseño y los nuevos materiales como estrategia de diferenciación. IX Congreso Latinoamericano de Enseñanza del Diseño. Universidad de Palermo, llevado a cabo en C.A.B.A, Argentina.

CAIP (Cámara Argentina de la Industria Plástica). (2016). Prohibición de bolsas plásticas. Rescatado de http://caip.org.ar/resolucion-de-la-ciudad-de-buenos-aires-prohibicionde-bolsas-plasticas/

Canetti, R., Bazoberri, J. (octubre de 2018). El diseño y los nuevos materiales como estrategia de diferenciación. IX Congreso Latinoamericano de Enseñanza del Diseño. Universidad Nacional de Cordoba, llevado a cabo en Córdoba, Argentina. 
Ellen MacArthur Foundation. (2013). Towards the Circular Economy, vol. 2. Rescatado de https://www.ellenmacarthurfoundation.org/publications/towards-the-circular-economyvol-2-opportunities-for-the-consumer-goods-sector

Etzkowitz, H. Leydesdorff, L. (2000). The dynamics of innovation from national systems and "mode 2" to a Triple Helix of University-Industry government relations. Research Policy, volumen (29), 109-123.

Galán, B. (2011). Innovación y pensamiento de Diseño, Foro de Innovación de las Américas. Ministerio de la Industria de Uruguay, Montevideo. Rescatado de https://www.youtube. com/watch?v=yTjDNu6kqoM

Gaines, S. (2014). "Reimaging Environmental Law for the 21st Century", Environmental Law Reporter, volumen (44), 10188-10215, 10213/14.

García, S. (2009). Referencias históricas y evolución de los plásticos. Historia de los polímeros. Revista Iberoamericana de Polímeros, volumen (10), 71 - 80.

Hassenzahl, M. (2010). Experience design: Technology for all the right reasons. Essen, Alemania: Morgan \& Claypool.

Jordan, P.W. (2000). Designing Pleasurable Products: An introduction to the New Human Factors. Londres, Reino Unido: Taylor and Francis.

Karana, E., Barati, B., Rognoli, V., van der Laan, A. Z. (2015). Material Driven Design (MDD): A Method to Design for Material Experiences. Revista Ij Design. Volumen (9), 35-54.

Karana, E., Pedgley, O., y Rognoli, V. (2014). Materials experience: Fundamentals of materials and design. Oxford, Reino Unido: Butterworth-Heinemann.

Kuhn, T.S. (1971). La estructura de las revoluciones científicas. FCE, México: Serie Breviarios.

Manzini, E. (1986). La materia de la invención. Materiales y proyectos. Barcelona, España: Editorial CEAC.

MinCyT (Ministerio de Ciencia, Tecnología, e Innovación Productiva). (2014). Análisis del patentamiento argentino entre 2008 y 2012. Rescatado de http://www.mincyt.gob.ar/ informes/analisis-del-patentamiento-argentino-entre-2008-y-2012-10667

Miodownik, M. A. (2007). Toward designing new sensoaesthetic materials. Revista Pure and Applied Chemistry, volumen (79), 1635 - 1641.

Moggridge, G.D., y Cussler E. L. (2000). An introduction to chemical product design. Revista Trans IChemE, volumen 78, 5-11.

Mulder, K., Ferrer, D., Van Lente, H. (2011). What is Sustainable Technology. Sheffield, Reino Unido: Greenleaf Publishing.

Natenzon, C. (2003). ¿Qué es la ciencia posnormal?. Escenarios Alternativos. Rescatado de http://www.escenariosalternativos.org/default.asp?seccion=escenarios3\&subseccion=e scenarios $3 \&$ nota $=1258$

OMPI (Organización Mundial de la Propiedad Intelectual). (2016). Datos y cifras de la OMPI sobre la P.i en 2016. Ginebra. Suiza. Rescatado de: http://www.wipo.int/edocs/ pubdocs/es/wipo_pub_943_2016.pdf

Pilar Productivo. (2018). Alerta de la industria plástica por las prohibiciones gubernamentales. noviembre 09, 2018, de Pilar Productivo Rescatado de http://www.pilarproductivo.com. ar/alerta-de-la-industria-plastica-por-las-prohibiciones-gubernamentales/

Sábato, J. Botana, N. (1968). La ciencia y la tecnología en el desarrollo futuro en América Latina. Revista de la integración, volumen (3),15-36. 
Sosa, A. (2013). Los plásticos: materiales a la medida. ¿Qué Ves?: Revista de divulgación de la ciencia de la UNAM, volumen (43), 22 - 25.

UNESCO (United Nations Educational, Scientific and Cultural Organization). (2015). Informe de la UNESCO sobre la ciencia. Hacia 2030. Resumen. Paris. Francia. Recuperado de https://en.unesco.org/unesco_science_report

van Der Lugt, P. (2008). Design interventions for stimulating bamboo commercialization: Dutch design meets bamboo as a replicable model (Tesis Doctoral). Universidad Tecnológica de Delft, Delft. Holanda.

van Kesteren IEH., y Kandachar P.V. (2004). Commercialization of new materials in consumer goods. Futureground, Proceedings of the Design Research Society International Conference 2004, 1-14.

Verganti, R. (2009). Design-driven innovation: Changing the rules of competition by radically innovating what things mean. Boston, Estados Unidos: Harvard Business Press.

Westerlund, S. (2008). Theory for Sustainable Development Towards or Against? Sustainable Development in International and National Law, volumen (53), 48-65.

\begin{abstract}
Improving the communication of Industrial Design with Materials Science represents a necessary action not only in terms of the provision of technical information intelligible to the field of design, but in its direct intervention in the applied research of new materials. The current socio-economic profile requires companies to resolve products with increasingly specific sensations and with greater respect for the environment. The regional application of the four-helix model with the incorporation of the user experience has advantages in the interrelation of interdisciplinary groups and fundamentally focuses attention on regional socio-environmental problems.
\end{abstract}

Keywords: Materials - Industrial Design - Circular Economy - Quadruple Helix Model User Experience - Innovation

Resumo: Melhorar a comunicação do Design com a Ciência dos Materiais representa uma ação necessária não so em termos de fornecimento de informação técnica inteligível ao campo do design, senão também na sua intervenção direta na pesquisa aplicada de novos materiais. $\mathrm{O}$ atual perfil socioeconômico exige as empresas resolver produtos com sensações cada vez mais específicas e com maior respeito pelo meio ambiente. A aplicação regional do modelo de quatro hélices com a incorporação da experiência do usuário tem vantagens na inter-relação de grupos interdisciplinares e enfoca fundamentalmente a atenção nos problemas socioambientais regionais.

Palavras chave: Materiais - Desenho Industrial - Economia Circular - Modelo Hélice Quádruplo - Experiência do Usuário - Inovação

[Las traducciones de los abstracts fueron supervisadas por el autor de cada artículo] 